\title{
Propuesta para la Elaboración de un Código de Etica del Estudiante de Medicina
}

\author{
ETHEL BAZAN \\ Departamento de Psiquiatria. Facullad de Medicina. - U.N.M.S.M.
}

\begin{abstract}
RESUMEN:
El autor y un grupo de alumnos presentan este docimento, con carácter de propuesta, el que consta de 38 artículos y procura aportar elementos en la formulación de un código que oriente la conducta (lel estudiante en relación a sí mismo, a sus profesores, compañeros, familiares y los pacientes (que participan en sll etapa formativa.
\end{abstract}

Palabrax clave: Etica, Estudiante de medicina, denntología.

\section{PROPOSAL FOR THE ELABORATION OF A MEDICAL STUDENT CODE OF ETHICS SUMMARY}

The author and a group of students propose this document consisting of 38 articles aimed to give the elements for the elaboration of a code that guide the conduct of the students in relation to themselves, their teachers, colleagues, relatives and patients that participate in their formative period.

Key words: Ethics, Student-medical, deontology.

\section{INTRODUCCION}

El alltor y su grupo de alumnos de seminario del curso de Elica Médica, en sesiones sucesivas elaboraron un Código de Etica del estudiante de Medicina. Se tuvo como base el Código de Etica y Deontología Médica del Colegio Médico clel Perú.

La Deuntología en particular es referida a una profesión o situación. El estudiante de medicina, se halla en una «situación» y por lo lanto necesita hormas mílimas mientras se forma como médico.

El grupo muy motivado y con la orienlación del autor, trabajó en varials sesiones cl código, que trata de resumir la conducta del estudiante, clescle el primer día de clases en la Escuela de Medicina Humana hasta su graicluación como médico.

\section{ASPLCTOS GENERALES}

El estudiante de medicina humana ingresa el primer día de clases con «aparente» morivación de ualiviar el dulor del ser humano y curar la enfermedad». Esta es la respuesta general.

Correspondencia:

D): Ethel Celestino Buzain Vidal

Faculsad de Medicina. U.N.M.S.M.

Av: Gran 755 Lima 1- Perá pero a no dudarlo, exísten orras motivaciones no reveladias como: provenir de una familia donde hay médicos: mejorar el status personal y familiar hasta aspectos de bienestar lindantes con mayores ingresos económicos, una motivación crematística. Merecería encuestar anónimamente a los recién ingresados pala descubrir detalles sobre estos aspectos.

Nos encontramos actualmente ante grandes avances tecnelógicos en la medicina, al punto en que es la máquina, el instrumento, el aparato, el exanen auxiliar y la compatadiora. los que rigen un diagnóslico y tratamiento: 10 que determina un aunento de la "deshumanización» del aclo médico, condición que cualquiera puede advertir por la probiferación de la publicidad de locto tipo. Una mercantilización de la medicinas.

La Facultad cle Medicina Humana de San Fernando de la Universidad Nacional Mayor de San Marcos, ha incorporaclo Elica y Deontología Médica como curso obligatorio para los estudialstes de medicina hace más de 3 años. en los últimos semestres de pre grado y, hace un año se dicla en el primer año (je pre-grado de estudios. Ajuste may importante y necesario.

El estudiante al ingresar: trae una serie de conductas y esililos de vida del adolescente. Durante sulormación. en el diario vivir como cstudiante. va gozando o sulfiendo. de la conducla de sus profesores y otos médicos. muchas veces olsecrvanclo cicras condirctas que se alcjan de lá ética. 


\section{PROPUESTA}

\section{Introducción}

La medicina es una profesión de servicio, desda este punto de vista el estudiante gue intentá ingrexar a sus filas astume la obligación de comportarse de acuerdo con sus más elevados ideales. El respeto entre la vida y la persona humana son la esencia espiritual de estos ideales que mantienen su legítima vigencia en nuestro quelacer formativo de cacla día, como auténtico tributo a nuestros maestros, a nuestra Alma Mater y al prọ́imo

Los principios éricos que condicional y gobiernan la conducta de los estudiantes de medicina deheráll distinguirse nor la singular nobleza de sus iduales, con proyección social, busadas lundamentalmente en la búsqueda de mejores condiciones du salud y vida para todos. Mejor calidad de vida.

En el atán de cumplir con estos altos ideales se buscalá asimilar los progresos cicntíticos más actualizados con la abuegada colaboración de sus maestros y cultores, tratando de desempeñar una importante función de modelo, orientador: conductor y guía para sus semejantes.

Esta «Propuesta para la elaboración de un Código de Etica del Estudiante de Medicina) constituye en el conjunto de preceptos de carácter moral que intenta asegurar una conducta hongorable, bonesta y ejemplar en su formación cultural, científica y espiritual del estudiante de medicina.

\section{Délheres Conmigo Mismo}

1. Prometo dedicarme a mi formación como médico integral y no permitiré que pasiones individuales y/o colectivas me desvíen de mi vocación.

2. Procuraré ser un estudiante modelo aplicando en mi los conocimientos de prevención primaria, secundaria, terciaria. Crear en mi un estilo de vida ejemplar.

3. Ocuparé mi tiempo fundamentalmente en el estudio, cumplimiento de todas mis tareas como estudiante, participando en todas las actividades educativas, seminarios, talleres, prác. licids, investigación y otros.

4. Mantendré una conducta puntual, disciplinada, y exacta en todos mis compromisos.

5. Me ayuclaré a mi mismo y a mis condiscípulos, familiares y pacientes que pudiera entrevistar.

6. Seré respetuoso en todos mis actos y haré que se me respete.

7. No ejerceré la medicina en forma jlegal, salvo las acciones de primeros auxilios, siempre que esté preparado.

8. Recordaré que la mejor fuente de aprendizaje es la observación integral y respetuosa de los pacientes con la guía de los maestros y libros actualizados.

9. Recordaré que la medicina integral no solo se aprende dentro de las aulas, sino en todas las circunstancias de la vida.

\section{Deleres para con Mis Maestros}

10. Respelaré a mis maestros, isí como espero que me icspeten mis alumnos cuando sed decente o múdico cojunplast.

11. Ororgáré a mis maestros la gratilud y consideración que merecen.
12 Seré colerante con las limitaciones: elrures y equivocaciones que pueda descubrir en mis maestros. De ser pusihle. respoIuosamente y a solas podré dialogar con ellos: evirando conduclas públicas que afectén a los maestrus.

13. Aprenderé y segruiré los mejores modelos que encuentre en mis macsirus

14. Conoceré y haré conocer el Código de Elica y Deontologéra del Colegio Médico del Perú.

\section{Deberes para con mis Compañeros}

15. Promoveré la reunión, fraternidad y alecon de hurinanos entre inis condiscípulus

16. Cumpliré y velaré porcjue se cumplan les precenlus monules y éticos entre mis compañeros.

17. Compartiré y haré que se compartan los conocimientos arlquiridos. inás actualizados, en el campo de la medicina.

18. Respetaré las ideds, creaciones y aporles de mis compañeros. reconociendo su autoría.

\section{Deleres para con los Pacientes}

19. Guardaré el más profundo respeco y consideración a los pacientes en todas las elapas de mi formación y después de ella.

20. Guardaré y haré que se guarde secrelo de los pacientes que entreviste durante mi formación y después de clla.

21. En la medida de mis posihilidades ayudaré: orientarté y seré suporte de los pacientes yue vea.

22. Suré respetuoso de la privacidad, el decoro. e idáas de los pacientes.

23. No haré distinciún de sexo, raza, rango social, filiación política, ni orientación sexual de los pacientes

24. Me pondré en la situación de los pacientes en cllalquice circunstancia, estableciendo la empatia necesaria.

25. Al ver pacientes los tratare como ine glistalia que me traten a mí o a un familiar cercano

26. No utilizaré mis conocimientos como medis de ohtener beneficios o favores que redunden en perjuicio moral o material de los pacientes.

\section{Deleres hacia los Familiares}

27. Recordaré que al ver pacientes veo familias, a los que debo toda consideración y respetn: pero la intormación directa de los pacientes es obligación del mécico tratante. por lo ranyo. como alumno guardaré rescrvas.

28. No aceptaré ni permitrié actitudes de fanniliares o allęados que puedan acarrear peligro o daño al pacienlc.

29. Con mis familiares, 10 defraudaré las expectativas de quienes con sacrificio ma dán apoyo.

\section{Deberes hacia la Comunidad}

30. Parliciparé permanentemente en acciones que promuevan la salud comunicaria.

31. Vejaré y lucharé pos que el medio amhience proceja al indivi|ा।

32. Respelaré los valores culturales de la cosmunidad. sills coslunhres y exilks de vila. si csios soll nocivos, ss mi deher educarlos: 
33. Scré educador en salud sin reservals ni eqoísmos.

it. No abandonaré a yaien acuda a mi por ayuda.

\section{Deberes hacia la Patria}

3.5. Ejerceré fielmente en la palria que permile mi formación y assí pagaré tributo al esilucrzo realizado.

\section{Deberex hacia mi Alma Mater}

36. Recordaré que mi Comación la debo a mi ALMA MATER y recordaré la obligación mojal de no defraudarla, siendo un huen médico.

37. Velaré por el cuiclado, buen funcionaniento y vigencia cienlifica de uni Alma Matcr.

3.8. Estaré dispuesto a apoyalr a mi alma mater ell cualyuicr circunstancia y en la medicla sle mis posibilidades.

\section{DISCUSION}

«El hombre está destinado a vivir en un medio social y esa vida es inseparable de la rełación con los otros hombres, teniendo por ello que aceplas la organización social, haciendo frence a ciertos principios y resipetándolos con el objeto de no perjudicarse ni perjudicar a los demás, aceplar que sul conducta está a lo que los otros aceptan lo que condiciona la paz y la garantía del medio donde vives. Eslasi afirmaciones de Baruk que incluimos en la Texis sobre la aplicación del Tesil de Tsedek justamen-

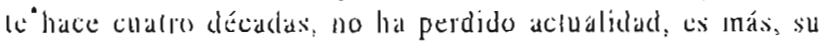
vigencia es mayor en los momentos acluales de acilerclo a la evolución de la medicina. Un grupo de estudiantes de medicina con un esfuerzo extraordinario de trabajo en equipo, con muchas reuniones formales coll el docente y otras entre ellos mismos: concretaron el Código con 38 artículos.

Como se aprecia. no se incluye sanciones por pretende FORMACION más que conocimiento, información, calificación o cistigo.

Lus aspecios cenlrales son muchos, desde la introducción del Código. cuyo texio fue elaborado por un responsable apoyado por olros compañeros y ha sido incluido en su versión original, espontáned. con lll contenido muy valioso y con una redacción ejomplar.

Los primeros 9 artículos sun originales, no sabenos de otros Códligos que cumiencen con DEBERES CONSIGO MISMO. con In intento de orientar en lo homalivo, una evaluación de la aulvestima. con reglas precisas que al ser cumplidas serán la hase de un MODELO DE ESTUDIANTE DE MEDICINA. Cada uno de los arlículos pueden ser desarrollados en amplitud, nos detemos en alorunos centrales, así en el attículo 2, donde el estudiante no solamente sie debe limilar al conocimiento. la incormación. siino descubriz en el mismo hábitos y conduclas que debc cambial: un inicio de una MENTALIDAD DE SALUD. Un ordenamicnlo de las costumbris y hábitos de) adolescente (muwhos de ellos a lodos). con conciencia clara de ser ya MODELO, si se silúan ell el conlexio de otros estudiantes de profesioIts diferentus. Dice el Código lextualmenle «CREAR EN MI UN ESTILO DE VIDA EJEMPLAR».
El art. 4 es un mandako que trala de camhiar condacias negativas de vida cultaral. Las normas élicas que concinliall apuntan a evitar la autofilia o narcicismo.

La ayuda inutua, germen di real servicio a los demás se extiende en forma natural al doliente: al enlermo. sin ol vidar las actitudes pretontivas que explícilamente se señalan en el rubro 2.

Diría: que estos primeros mandamientos. son el primer paso formativo, el inicio de un médico ejemplar que se silúe yá cuando profexional. en un lugar digno en la sociedad.

Es momento de recordar que la pre-adulexcencia. la adolescencia mediana y tardía no esscapan al confliclo gencracional. la turbulenciia homonal sexual, la afirmación de la identidad del yo. Deside exta perspectiva el Código no solumente es úryil pááa

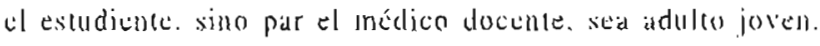
maduro o en clapas de la senceptud: pires. compromesc al maestro, su inleligente comprensión: quicill. al veces: no es concientl de su allura de MODELO SOCIAL. uncho nenos de MODELO DE SUS DISCÍPULOS.

Los artículos $10^{\circ}$ - $14^{\circ}$ sitúan al extudiante en la relación diádica MAESTRO-ALUMNO, las reglas son de un humanismo durante la experiencia de alumno. proyectándose como luturo Cormadol de médicos del mañana. Dicho humanismo se eleva con normas de comportamiento frente al prolesor. la tolerancia en las limitacioncs o alcances. sus delectos al mismo licmpo yuc oriemea a una aproximación saludable con su mas.stro. Al mismo ticmpo le permite determinar la callidad de uno 0 más docentes. modelos evidentes, evjlando contagiarse de alelitudes a conductas de otros que no lo son.

Cuando nos delenemos en los artículos $15^{\circ}-18^{\circ}$. consideramos que se inserlan semillas de unión. respeto mutuo y permitan que el futuro respete la AUTORIA. (an poco desarrullada en muchos méclicos.

Merece consideración expecial LOS DEBERES CON LOS PACIENTES, desde el 19 al 26 articulos: pues, a diferencid de otros países, en el Perú, el alumno se ponte en conlacto con enfermos y familiares, hace visitas domiciliarias. clesele los primeros ciclos, especílicamente: cuankio con las prácticas del carso de Ciencias del Comportamiento en Medicina. Esic cursa. que permite la premera colrevista formal. eron el partínte en cama. en consulta externda, visila domiciliariat debidanente orientada, es la primera aproximación clcl estudiante al enfermo. Los responsables directos de las primeras prácticás. ohservamos y senlimos las emociones de los alumnos. gutándolos para dsumir veracidad, realidad, en el inicio de la carrera y evilankio conducdas díadicas o duales: 4 no compromelerse eon aclitudes de lamiliatridad, ni tomar distancials duales naturales en otros contactos humanos. Posteriormente lay cursos como semiología. clínica, en que los contaclos persona-persona son mais frecuen-

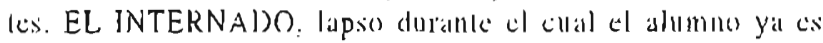
responsable de actos médicos, atscisoraclo por médicos en pléno éjescicior. ex ya la ligura de un médico. es el nacicute probexional, es el parto de un nucvo galeno. EL SERUMS ex el nacimicn(1) del Inéclico. al servicio del doliente. Mucho más si cijace en zonas donde no ha llegado la cohertura en salud. 
LOS DEBERES hacia los familiares, art. 27 al 29, son subrayados como una extensión de los precedentes y enlaza a la propia familia del estudiante; pues, como persona es parte de un núcleo, con sus virtudes y defectos, con salud y enfermedad, con problemas o sin ellos. No puede pues el estudiante ignorar su propia familia, es más, respetuosamente con el ejemplo, ser lambién modelo para hermanos y utros familiares.

El Código no olvida los deberes HACLA LA COMUNIDAD doncle se halle el estudiante o profesional, enfatiza en desariollar una conducra de EDUCADOR, al mismo tiempo que destaca actualizar la revolución en salud, que es la defensa de un medio ambiente saludable, la cultura en sálud y el respeto a los valores culturales.

Mucho se babla del MEDICO QUE NECESITA EL PERU, no se hasta que punto el diseño curricular permite este enfoque, lo que si es evidente, la emigración de muchos médicos a otros países, una fuga de talentos. Los DEBERES HACIA LA PATRIA señalan la obligación de ejercer en su terruño, en su patria. Es una obligación moral, no es un mandato imperativo; pero, mueve en reflexionar en la posibilidad de que los médicos que emigran, con un marco legal ha establecerse, reembolse al Perú el costo de su formación, desde el kindergarten, secundaria y superior; una forma, si bien no completa, que permita a la Uñiversidad, al País, aunque sea en lo mínimo, pagar los 160 más años que se ha utilizado en el producto, la formación de un MEDICO. Estas reflexiones no son originales, hay paises donde no solamente el alumno, estudia gratuitamenle, sino se le paga: pero, cuando ejerce la profesión, está obligado a pagar los costos (Suecia).

El claustro materno, la ALMA MATER, el lugar donde se concibe, desarrolla y nace el médico, necesita ser señalada y destacada. Es un aspecto ignorado por muchos, es valorar lo nuestro, es cuidar de ella, es expresar gratitud, es defenderla, es volver a ella y contribuir a que el claustro que cobijó a los MEDICOS FORMADOS sea cada vez mejor, es la obligación moral de apoyarla, no solamente con el sano orgullo de haber pertenecido a ella, sino, nutrirla con nuestra propia contribución perso- nal a lo menos, con aportes y donaciones de acuerdo a las posibilidades de lOS SAN FERNANDINOS. SAN MARQUINOS.

\section{CONCLUSIONES}

1. El presente trabajo es producto del esfuerzo conjunto docente y alumno.

2. Pretender ser tomado en cuenta y de ser posible, ser considerado como documento a ser cumplido, hasta cuando se elabore el Código definitivo.

3. La actividad humana no es perfecta, creo que la proputesta no escapa a ello, por lo tanto, puede ser mejorarta.

4. Cada una de los artículos puede ser motivo de una amplia y variada discusión en los diferentes talleres de los cursos de Etica y en todo momento en que sea necesario apoyarse para la formación en el Claustro San Fernandino.

5. Consideramos que la propuesta no sólo debe ser discutida y estudiada por los estudiantes, sino, por los docentes y médicos yá formados.

\section{AGRADECIMIENTO}

En la elaboración del presente documente colaboraron los siguientes alumnos: María Valdez Iglesias, Vera Nonoskque, Nancy Inostroza, Trujillo Salvador, Máximo Vega, César Villa, Luis Viltar, Davis Zambrano, Guido Zamudio, Fernando Zarzusa, Richard Zegarra (Grupo de Seminario del curso de Etica).

\section{BIBLIOGRAFIA}

1) Código de Etica y Deontología del Colegio Médico del Perú. Decreto Ley 1723-01-01-1070

2) Escardó Florencio. Etica Médica. 1970. Editorial Ateneo Buenos Aires. Argencina.

3) Bazán Vidal Ethel Celestino. Test de Tsedek en Esquizofrénicos (Tsedek, medición del Componence Etico de la Personalidad). Tesis de Bachiller en Medicina. 1955

4) Manual de Psiquiatría Hunlyerto Rotondo. 1991. Edicoses Perales A.; Zambrano M.; Vásquez Caicedo G.; Mendoza A.

5) Freedmamn, A. Kaplan. Sadock B. Tratado de Psiquiatría. Editorial Salvat. 